\title{
Aplicação de RNAs para Predição de Índice de Viscosidade: uma avaliação da qualidade de biodiesel
}

\author{
Raquel Machado de Sousa ${ }^{1}$, Sofiane Labidi ${ }^{2}$, Jailson Nunes Leocadio ${ }^{1}$ \\ ${ }^{1}$ Laboratório de Automação Agrícola (LAA) - Universidade de São Paulo (USP) \\ São Paulo - SP - Brasil \\ ${ }^{2}$ Laboratório de Sistemas Inteligentes (LSI) - Universidade Federal do Maranhão \\ São Luís - MA - Brasil \\ \{rachel.msousanet, jailsonleocadio\}@gmail.com, labidi@dee.ufma,br
}

\begin{abstract}
To measure the biodiesel quality in Brazil, several parameters are established for this purpose. Some of them, such as viscosity, iodine number, cetane number, density are important because they characterize important functions of how biodiesel will react in the engines. In this study, Artificial Neural Networks (ANNs) were used to predict the viscosity value of biodiesel. For this purpose, 13 compounds of fatty acid esters were used as input to the ANNs, with several feedforward convergence algorithms having viscosity indexes as output from the networks.
\end{abstract}

Resumo. Para mensurar a qualidade do biodiesel no Brasil, vários parâmetros são estabelecidos. Alguns deles como viscosidade, índice de iodo, número de cetano, densidade são importantes pois caracterizam funções importantes de como o biodiesel irá reagir no motores. Neste estudo, foram usadas Redes Neurais Artificiais (RNAs) para predizer o índice de viscosidade do biodiesel. Para este fim foram utilizadas 13 compostos de esteres de ácidos graxos como entrada para as RNAs, com vários algoritmos de convergência do tipo feedforward tendo como saída das redes os índices de viscosidade.

\section{Introdução}

A corrida mundial por alternativas limpas de energia, ou seja, aquelas geradas a partir de recursos renováveis e que não agravam os problemas de emissão de gases poluentes na atmosfera, fez com que vários países passassem a buscar alternativas e a realizar pesquisas direcionadas ao consumo de combustíveis de maneira sustentável. O biodiesel, por ser uma fonte energética oriunda de biomassa, é capaz de contribuir, com sustentabilidade, para suprir essa demanda mundial.

O biodiesel apresenta diversas propriedades que estão relacionadas aos contaminantes da matéria-prima, às condições de armazenamento e, principalmente, às estruturas moleculares que o compõem, que são utilizadas para identificar a qualidade dele. Para assegurar uma combustão eficiente e qualidade nas emissões, bem como a segurança no transporte e manuseio do biodiesel, a Agência Nacional do Petróleo, Gás Natural e Biocombustíveis (ANP) instituiu, por meio da Resolução ${ }^{0} 14$ de 2012 (atualizada para ${ }^{0}$ 45 em 2014), parâmetros de qualidade e especificações para o biocombustível.

As propriedades inerentes à composição, como número de cetano, densidade, viscosidade, número de iodo, entre outros, estão diretamente relacionadas ao percentual de 
cada éster alquílico que compõe a matéria-prima (óleo vegetal ou gordura animal). Estudos sobre a influência da composição química do biodiesel nas suas propriedades de qualidade têm sido demonstrada por meio de pesquisas que ressaltam que as propriedades dos triglicerídios e do combustível biodiesel são determinadas pelas quantidades de cada ácido graxo que se encontram presentes em suas moléculas [Ramos et al. 2009].

Diferentes técnicas de previsão de propriedades de qualidade como índice de iodo, viscosidade cinemática, densidade, número de cetano, entre outros, são focos de análises. Algumas abordagens usam modelos empíricos e estatísticos [Allen et al. 1999, Ramírez-Verduzco et al. 2012, García et al. 2013, Chavarria-Hernandez and Pacheco-Catalán 2014, Freitas et al. 2014], além de técnicas que utilizam espectroscopia de infravermelho próximo (NIR) para determinar as propriedades do biodiesel [Baptista et al. 2008, Adewale et al. 2014]. As Redes Neurais Artificiais (RNAs), por sua vez, têm sido aplicadas em várias áreas da engenharia e domínios. Em relação às pesquisas relacionadas com controle de qualidade de combustíveis e biocombustíveis, essas redes têm sido empregadas nos mais variados contextos [Çay et al. 2013, Sharon et al. 2012, Piloto-Rodríguez et al. 2013, Saldana et al. 2012, Rezaee et al. 2014].

A previsão da viscosidade cinemática tem recebido atenção considerável na literatura, pois é uma das propriedades mais importantes que afetam a utilização do biodiesel como combustível [Allen et al. 1999]. Tanto a viscosidade do óleo e seu éster alquílico derivado podem ser utilizados para monitorar a qualidade do biodiesel [Allen et al. 1999]. É uma propriedade que possui relação direta com o processo de injeção dos motores, a viscosidade do biodiesel aumenta com o comprimento da cadeia carbônica e com o grau de saturação [Knothe et al. 2010]. A norma EN 14214 (EN ISO 3104) estabelece um intervalo aceitável de 3,5 a 5,0 $\mathrm{mm} 2$ /s, ASTM D6751 (D 445) permite um intervalo de 1,9 a $6,0 \mathrm{~mm} 2 / \mathrm{s}$ e a ANP com limite aceitável de 3,0 a $6,0 \mathrm{~mm} 2 / \mathrm{s}$. A ANP além dos métodos analíticos citados recomenda também o método ABNT NBR 10441 [Dantas 2006].

A pesquisa de [Jahirul et al. 2013] utilizou redes neurais artificiais para predição da viscosidade cinemática a partir da influência da temperatura. O trabalho utilizou 204 amostras com 23 entradas incluindo temperatura e composição química do biodiesel e a propriedade da viscosidade cinemática como saída. As RNAs possuiam uma camada oculta variando de 23 a 46 neurônios e as redes foram treinadas somente com o algoritmo Levenberg Marquardt. A melhor performance obtida foi com a topologia (23:28:1) com índice de determinação $(\mathrm{R})$ de 0,97 ; por outro lado, a acurácia de predição do modelo pode ser melhorada por meio do aumento do intervalo de treinamentos e conjunto de dados.

[Meng et al. 2014] também conduziu seu trabalho com predição da viscosidade cinemática. Nesse estudo, uma RNA foi desenvolvida para prever a viscosidade cinemática do biodiesel à temperatura de $313 \mathrm{~K}$, com os dados experimentais de 105 amostras de biodiesel recolhidas a partir da literatura. O método utilizou na RNA apenas as frações de massa de 19 ésteres metílicos de ácidos graxos como entradas. Dois métodos previamente descritos baseados em equações empíricas foram comparados com os resultados da RNA. Os resultados indicaram que o método com RNA proposto é capaz de prever a viscosidade cinemática a $313 \mathrm{~K}$ do biodiesel com erro quadrático médio (MSE) de 0.0099. No geral, o método com RNA demonstrou uma melhor precisão para a previsão da viscosidade 
cinemática biodiesel com maior coeficiente de correlação de 0.9774 .

Este trabalho quer contribuir com os estudos de RNAs para predição dos índices de viscosidade por meio do treinamento de redes feedforward, pela avaliação de diferentes algoritmos de convergência e obtenção de um índice de correlação de predição que contribui para a utilização de RNAs para predição dos parâmetros de qualidade de biodiesel. O trabalho está organizado da seguinte forma: Na seção 2 é apresentado os dados experimentais utilizados. Na seção 3 é descrita a metodologia de desenvolvimento das RNAs. Na seção 4 é mostrado os resultados e discussões e, por fim, a seção 5 traz as considerações finais sobre a pesquisa.

\section{Dados Experimentais}

Neste estudo, os dados utilizados para treinamento, validação e teste das redes foram reunidos da literatura [Santos 2008, Dantas 2006, Candeia 2008, Padhi 2010, Sanford et al. 2009, Moser 2009, Hoekman et al. 2012, Kivevele et al. 2011, Nogueira 2010] e outros cedidos pelo Laboratório de Pesquisa em Química Analítica (LPQA) da Universidade Federal do Maranhão (UFMA). Esses dados são referentes à composição e as suas análises físico-químicas de amostras de biodiesel. Um conjunto de 98 amostras com 13 ésteres de entrada e saída representada pela viscosidade.

Pode-se verificar pela Figura 1 a distribuição de cada tipo de ésteres de ácidos graxos nas amostras de biodiesel coletadas e a predominância dos ésteres, como o ácido oleico (C18:1), ácido linoleico (C18:2), ácido laurico (C12:0) e ácido palmítico (C16:0). A descrição para a composição dos esteres neste trabalho (XX:X) é sobre a informação do número de carbonos $(\mathrm{XX})$ e o número da direita representa o número de insaturações da molécula. As amostras de biodiesel são derivadas de vários tipos de óleos e oleoginosas, entre elas, biodiesel metílico e etílico de soja, dendê, linhaça, colza, pinhão manso, moringa, babaçu, gordura vegetal de aves e algas; as diversas cores na figura 1 representam os diversos tipos citados.

\section{Metodologia de Desenvolvimento de RNAs}

A construção de uma topologia de RNA considerada mais apropriada para resolver um problema específico é usualmente efetuada de forma empírica, pois tal dimensionamento depende, dentre outros fatores, do algoritmo de aprendizagem utilizado, da forma como as matrizes de pesos foram iniciadas, da complexidade do problema a ser mapeado, da disposição espacial das amostras e da qualidade do conjunto de treinamento disponível [Da Silva et al. 2010].

Em termos práticos, no processo de construção de RNAs é possível identificar duas fases principais: fase de treinamento e fase de operação. Cada fase possui as seguintes atividades.

- Fase de treinamento:

- Selecionar padrões de treinamento e teste;

- Pré-processar padrões(normalizar os dados);

- Definir topologias candidatas;

- Treinar topologias candidatas;

- Escolher melhor topologia; 


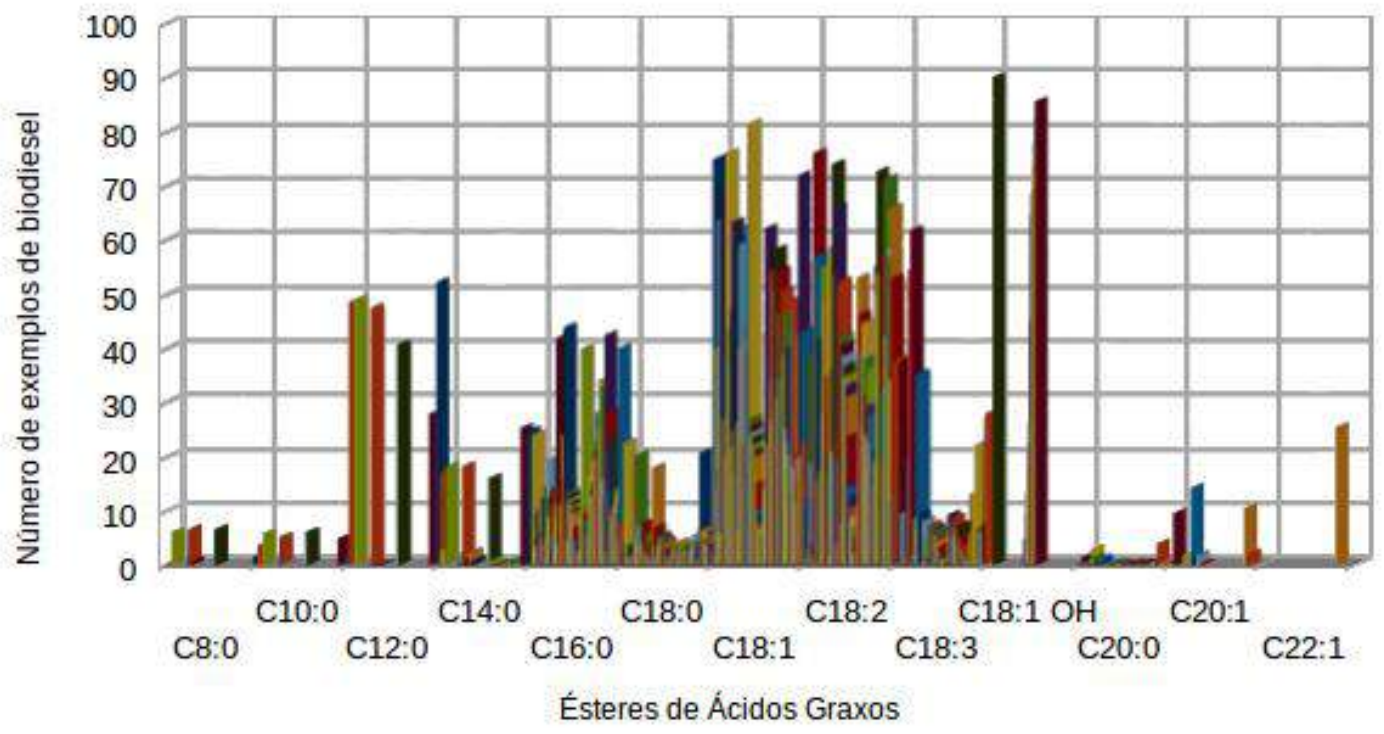

Figura 1. Quantidade de esteres predominantes nas amostras de biodiesel

- Fase de operação:

- Apresentar padrões de entrada;

- Pré-processar padrões (normalizar dados);

- Aplicar padrões nas entradas da rede treinada;

- Obter resultados das saídas da rede treinada;

- Pós-processar resultados (desnormalizar dados);

As RNAs usadas neste estudo foram do tipo feedforward backpropagation, que são uma das mais populares em vários domínios e baseadas no aprendizado supervisionado [Meng et al. 2014]. As redes foram configuradas com uma e duas camadas escondidas, com números de neurônios variando de 2 a 25 em cada camada. Vários algoritmos podem ser utilizados para otimizar arquiteturas de redes, destes foram selecionados 7 para treinamento, validação e teste: backpropagation padrão (gradiente descendente), backpropagation com momentum e taxa de aprendizado, BFGS quasi-newton, gradiente conjugado escalonado, resilient propagation, one step secante e Levenberg-Marquardt.

Os algoritmos selecionados foram divididos em duas categorias de estratégias de treinamento: métodos de primeira e de segunda ordem. Os métodos de primeira ordem são aqueles onde somente o gradiente local determina a direção da minimização do erro (gradiente descedente). Já os métodos de segunda ordem são considerados mais eficientes para fazer o treinamento de redes do tipo múltiplas camadas. Esses algoritmos recorrem a um rigor matemático baseado em modelos bem definidos de otimização não-linear não estendida, e não apresentam assim um vínculo natural com a inspiração biológica proposta inicialmente para RNAs. Podemos citar o método de Newton, que pode ser considerado como o método local básico que usa informações de segunda ordem, sua aplicação requer o cálculo da matriz de Hessian[Silva 1998].

Foram selecionadas também duas funções de ativação: função logística e função tangente hiperbólica. Pode-se visualizar na Tabela 1 a descrição dos principais parâmetros 
configurados.

Tabela 1. Configuração das topologias de redes simuladas.

\begin{tabular}{|c|c|}
\hline Parâmetros de treinamento & Valor \\
\hline Algoritmos & $\begin{array}{c}\text { Backpropagation padrão } \\
\text { Backpropagation com momentum e taxa de aprendizagem } \\
\text { BFGS Quase-Newton } \\
\text { Levenberg-Marquardt } \\
\text { Resilient-propagation } \\
\text { One-Step-Secant } \\
\text { Gradiente Conjugado Escalonado }\end{array}$ \\
\hline Função de ativação & $\begin{array}{ll}\text { Função tansig } \\
\text { Função logsig }\end{array}$ \\
\hline Número de camadas ocultas & 1 e 2 \\
\hline Função de desempenho & MSE \\
\hline Critério de generalização & $\begin{array}{l}\text { Validação cruzada em } k \text {-partições } \\
\text { e parada antecipada }\end{array}$ \\
\hline $\begin{array}{l}\text { Valor máximo de verificações } \\
\text { de parada antecipada }\end{array}$ & $6,10,15,20$ e 25 \\
\hline Número máximo de épocas & 10000 \\
\hline Taxa de aprendizagem & 0.01 \\
\hline Número de neurônios por camada & $2-25$ \\
\hline
\end{tabular}

Ao todo, 576 combinações de redes para cada algoritmo de treinamento foram testadas. Os dez melhores modelos de cada algoritmo foram salvas por meio do método de validação cruzada $(k$-fold) [Kohavi et al. 1995]. Além do critério de validação cruzada com $\mathrm{k}=11$ para escolha da melhor rede entre as candidatas, a técnica de treinamento com parada antecipada foi usada afim de detectar overfitting durante a fase de treinamento da rede.

A definição da quantidade de verificações de erros para parada antecipada foi obtida mediante testes das redes, com os seguintes valores de erros: 6, 10, 15, 20 e 25 . O cálculo do erro usado para mensurar o desempenho da rede foi o Erro Médio Quadrático ou Mean Squared Error (MSE), que interpreta a diferença entre o valor produzido pela rede, indicado por $\hat{y}_{i}$, e o valor real, $y_{i}$ [Meng et al. 2014]:

$$
M S E=\frac{1}{n} \sum_{i=1}^{n}\left(\hat{y}_{i}-y_{i}\right)^{2}
$$

Desse modo, 10 k-partes foram usadas para treinamento e validação; e a partição $k=11$ usada para teste. As entradas e saídas de dados para treinamento, validação e teste foram pré-processadas entre -1 e 1. A Regressão Linear (Linear Regression - $R$ ) e a Raiz Quadrada Média do Erro (Root Mean Square Error - RMSE) foram usadas para análise de desempenho. Os valores de R e RMSE são uma indicação de correlação entre as saídas preditas e as saídas reais da rede.

$$
\begin{gathered}
R=\alpha+\beta X_{i}+\epsilon_{i} \\
R M S E=\frac{\sqrt{\sum_{i=1}^{n}\left(\hat{y}_{i}-y_{i}\right)^{2}}}{n}
\end{gathered}
$$

Ainda em relação aos critérios de configuração das topologias de rede, os pesos da 
rede foram iniciados aleatoriamente, foi definido um limite de 10000 mil épocas (quantidade de ciclos) de treinamento para cada topologia e a taxa de aprendizagem no valor de 0,01 . No final das simulações as 10 melhores redes foram salvas.

\section{Resultados e Discussões}

Neste estudo foram utilizadas 98 amostras de ésteres de ácidos graxos para predição de viscosidade cinemática. Foram desenvolvidas e testadas 576 combinações para cada algoritmo para se obter as melhores topologias candidatas para predição de propriedades de qualidade do biodiesel. Os testes foram executados com a ferramenta Matlab Version 2013 b Neural Netwoks Toolbox.

\subsection{Considerações sobre o critério de parada antecipada}

O uso da validação cruzada com parada antecipada é uma importante etapa para evitar overfitting. Assim, a definição do número de verificações de erro é um ponto essencial e crítico no treinamento da rede. O Levenberg-Marquardt foi o algoritmo de convergência usado para teste dos valores de erros, com duas camadas ocultas e função de ativação tangente hiperbólica para averiguar qual valor produzia melhor performance na predição da viscosidade. As redes foram treinadas com separação de dados por amostra aleatória e por k-partições. Os resultados dos coeficientes de correlação obtidos das simulações para escolha do valor de parada antecipada são reportados na Tabela 2.

Tabela 2. Resultados dos testes para escolha do valor de parada antecipada para viscosidade.

\begin{tabular}{c|c|c|c|c|c}
\hline Configuração da Rede & Valor de parada antecipada & Treinamento - R & Validação - R & Teste - R & Separação dos dados \\
\hline $12-4$ & 6 & 0.99 & 0.88 & 0.97 & Aleatória \\
$5-10$ & 6 & 0.99 & 0.86 & 0.97 & k-partições \\
\hline $\mathbf{1 1 - 5}$ & $\mathbf{1 0}$ & $\mathbf{0 . 9 9}$ & $\mathbf{0 . 9 9}$ & $\mathbf{0 . 9 6}$ & Aleatória \\
$\mathbf{5 - 3}$ & $\mathbf{1 0}$ & $\mathbf{0 . 9 8}$ & $\mathbf{0 . 9 9}$ & $\mathbf{0 . 9 9}$ & k-partições \\
\hline $4-10$ & 15 & 0.99 & 0.98 & 0.70 & Aleatória \\
$8-12$ & 15 & 0.99 & 0.99 & 0.80 & k-partições \\
\hline $3-18$ & 20 & 0.99 & 0.99 & 0.83 & Aleatória \\
$4-5$ & 20 & 0.99 & 0.99 & 0.45 & k-partições \\
\hline $13-2$ & 25 & 0.98 & 0.99 & 0.83 & Aleatória \\
$6-7$ & 25 & 0.99 & 0.99 & 0.73 & k-partições \\
\hline
\end{tabular}

Dos resultados obtidos pode-se aferir que valores de parada antecipada muito baixos ou valores muito altos não permitem que a rede aprenda o suficiente para generalizar bem os dados de treinamento, validação e teste; assim, classificam incorretamente novos dados.

\subsection{Resultados da viscosidade cinemática}

A viscosidade é uma importante medida da resistência da vazão de um líquido associada à fricção ou atrito interno de uma parte do fluido que escoa sobre a outra, ocasionando formação de depósitos no motor. Quanto maior a viscosidade maior é a tendência do combustível causar problemas. Esta propriedade pode ser determinada por metodologias padronizadas como ASTM D445 ou a ISO 3104. Por sua vez, a análise da diferença da viscosidade entre o óleo de origem e o seu derivado aquiléster pode ser utilizada para monitorar o biodiesel [Knothe et al. 2010].

Pode-se inferir dos resultados obtidos de predição de viscosidade com redes de uma camada que o algoritmo Levenberg-Marquardt obteve o melhor coeficiente de 
correlação para rede com 8 neurônios na camada oculta. A regressão chegou a valores de 0,99 tanto para treinamento, validação e teste. O segundo melhor desempenho foi com o algoritmo BFGS que conseguiu generalizar com coeficientes de correlação de 0,99 para treinamento, 0,99 para validação e 0,96 para teste, que pode ser observado na Tabela 3. Além dos valores de regressão observados, o RMSE obtido dos algoritmos de treinamento testados ficaram abaixo de 1 .

Dos resultados obtidos de previsão de viscosidade com duas camadas, observouse que praticamente todos os algoritmos apresentaram um bom desempenho para generalização de dados novos. Exceto pelos algoritmos BFGS e resilient-propagation que obtiveram baixos desempenhos na fase de teste da rede, o que pode significar um overfitting do treinamento, em vista da quantidade de neurônios nas camadas ocultas, apesar da validação cruzada.

Pode-se comparar também o desempenho do backpropagation padrão, backpropagation com momentum e o Levenberg-Marquardt. Os três obtiveram coeficientes de correlação de 0,99 para treinamento, validação e teste da rede. Porém o que obteve menor número de neurônios foi o Levenberg-Marquardt com uma arquitetura 5-6, ou seja, 5 neurônios da primeira camada oculta e 6 neurônios na segunda camada oculta. Enquanto que o backpropagation padrão e com momentum precisaram de um número superior de neurônios, com arquiteturas de 22-9 e 20-8, respectivamente, para generalizar bem com esses coeficientes.

A Tabela 4 mostra todos os coeficientes obtidos para cada algoritmo na predição da viscosidade com duas camadas. Trabalhos relatados [Jahirul et al. 2013, Meng et al. 2014, Saldana et al. 2012] já demonstraram a habilidade do algoritmo Levenberg-Marquardt de predizer a propriedade viscosidade cinemática por meio de RNAs. Neste estudo, confirma-se essa habilidade também para a viscosidade, porém enquanto que alguns trabalhos relacionados chegaram a apenas 0.97 na predição dessas propriedades, aqui obtive-se índices de 0.99 de predição.
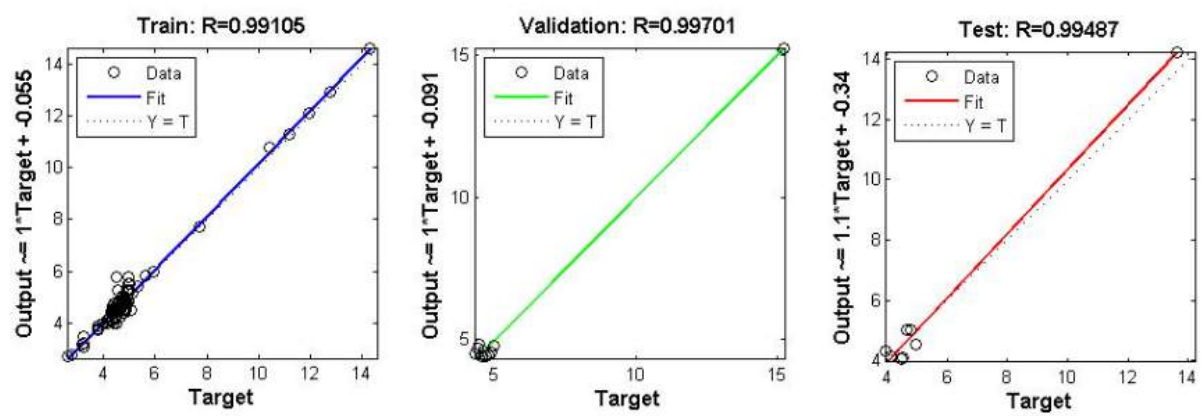

Figura 2. Coeficiente de correlação da viscosidade para Levenberg-Marquardt com uma camada

É preciso considerar também que os resultados obtidos para viscosidade realizados com uma camada oculta já são considerados suficientes de acordo com os testes. Esses resultados obtidos de 0.99 de predição, tanto para validação e teste do LevenbergMarquardt, podem ser observados na Figura2. Isso porque a finalidade do treinamento e técnicas de escolha de melhor topologia de rede, é encontrar a arquitetura que ofereça o 
melhor desempenho com menor custo computacional, ou seja, com menor quantidade de neurônios.

Tabela 3. Coeficientes de predição para viscosidade com redes de uma camada oculta.

\begin{tabular}{|c|c|c|c|c|c|c|c|c|}
\hline $\begin{array}{c}\text { Configuração } \\
\text { da Rede }\end{array}$ & $\begin{array}{c}\text { Algoritmos } \\
\text { de Treinamento }\end{array}$ & $\begin{array}{l}\text { Função de } \\
\text { Ativação }\end{array}$ & $\begin{array}{c}\text { Treinamento } \\
\text { R }\end{array}$ & RMSE & $\begin{array}{c}\text { Validação } \\
\text { R }\end{array}$ & RMSE & $\begin{array}{c}\text { Teste } \\
\mathrm{R}\end{array}$ & RMSE \\
\hline 13 & Backpropagation padrão & logsig & 0.98 & 0.33 & 0.99 & 0.44 & 0.94 & 0.35 \\
\hline 20 & $\begin{array}{c}\text { Backpropagation } \\
\text { com momentum e } \\
\text { taxa de aprendizagem }\end{array}$ & logsig & 0.98 & 0.4305 & 0.96 & 0.2979 & 0.3332 & 0.6893 \\
\hline 8 & BFGS Quase-Newton & logsig & 0.99 & 0.2791 & 0.99 & 0.3495 & 0.96 & 0.6685 \\
\hline 9 & $\begin{array}{c}\text { Gradiente Conjugado } \\
\text { Escalonado }\end{array}$ & logsig & 0.99 & 0.33 & 0.77 & 0.4405 & 0.97 & 0.1911 \\
\hline 22 & Resilient-propagation & logsig & 0.99 & 0.3186 & 0.41 & 0.5762 & 0.99 & 0.4966 \\
\hline 17 & One-Step-Secant & logsig & 0.98 & 0.3895 & 0.97 & 0.1785 & 0.96 & 0.5556 \\
\hline 8 & Levenberg-Marquardt & logsig & 0.99 & 0.276 & 0.99 & 0.2772 & 0.99 & 0.3803 \\
\hline
\end{tabular}

Tabela 4. Coeficientes de predição para viscosidade com redes duas camadas ocultas.

\begin{tabular}{|c|c|c|c|c|c|c|c|c|}
\hline $\begin{array}{l}\text { Configuração } \\
\text { da Rede }\end{array}$ & $\begin{array}{c}\text { Algoritmos } \\
\text { de Treinamento }\end{array}$ & $\begin{array}{l}\text { Função de } \\
\text { Ativação }\end{array}$ & $\begin{array}{c}\text { Treinamento } \\
\mathrm{R}\end{array}$ & RMSE & $\begin{array}{l}\text { Validação } \\
\text { R }\end{array}$ & RMSE & $\begin{array}{c}\text { Teste } \\
\mathrm{R}\end{array}$ & RMSE \\
\hline $22-9$ & Backpropagation padrão & logsig & 0.98 & 0.3388 & 0.99 & 0.3721 & 0.99 & 0.2694 \\
\hline $20-8$ & $\begin{array}{c}\text { Backpropagation } \\
\text { com momentum e } \\
\text { taxa de aprendizagem }\end{array}$ & tansig & 0.99 & 0.3159 & 0.99 & 0.5098 & 0.99 & 0.108 \\
\hline $11-12$ & BFGS Quase-Newton & $\operatorname{tansig}$ & 0.97 & 0.4619 & 0.99 & 0.2158 & 0.63 & 0.4493 \\
\hline $18-13$ & $\begin{array}{l}\text { Gradiente Conjugado } \\
\text { Escalonado }\end{array}$ & tansig & 0.99 & 0.3063 & 0.96 & 0.3698 & 0.98 & 0.4916 \\
\hline $21-4$ & Resilient-propagation & logsig & 0.98 & 0.316 & 0.99 & 0.418 & 0.32 & 0.3046 \\
\hline $10-13$ & One-Step-Secant & logsig & 0.98 & 0.342 & 0.94 & 0.2555 & 0.98 & 0.4765 \\
\hline $5-6$ & Levenberg-Marquardt & tansig & 0.99 & 0.1785 & 0.99 & 0.2252 & 0.99 & 0.418 \\
\hline
\end{tabular}

\section{Conclusão}

Da avaliação desses algoritmos, pode-se perceber que as RNAs são uma boa ferramenta para predição de parâmetros de qualidade do biodiesel. Os resultados demonstraram índices de correlação das melhores arquiteturas de redes para predição da viscosidade com índice de correlação de 0.99 tanto para treinamento, validação e teste.

Desse modo, considera-se que com a avaliação dos algoritmos é possível verificar a tendência da qualidade do biodiesel a partir de suas propriedades físico-químicas e assim reduzir tempo e custos na indústria de biocombustíveis, tendo em vista que alguns métodos de análises de biodiesel podem ser longos e complexos.

\section{Referências}

Adewale, P., Mba, O., Dumont, M.-J., Ngadi, M., and Cocciardi, R. (2014). Determination of the iodine value and the free fatty acid content of waste animal fat blends using FT-NIR. Vibrational Spectroscopy, 72(0):72-78.

Allen, C., Watts, K., Ackman, R., and Pegg, M. (1999). Predicting the viscosity of biodiesel fuels from their fatty acid ester composition. Fuel, 78(11):1319-1326.

Baptista, P., Felizardo, P., Menezes, J. C., and Neiva Correia, M. J. (2008). Multivariate near infrared spectroscopy models for predicting the iodine value, CFPP, kinematic 
viscosity at 40 degrees $C$ and density at 15 degrees $C$ of biodiesel. Talanta, 77(1):14451.

Candeia, R. A. (2008). Soybean Biodiesel: Synthesis, Degradation and Binary Mixtures. $\mathrm{PhD}$ thesis, Doctoral dissertation, D. Sc. Thesis. Federal University of Paraíba. Brazil. Pag. 138.

Çay, Y., Korkmaz, I., Çiçek, A., and Kara, F. (2013). Prediction of engine performance and exhaust emissions for gasoline and methanol using artificial neural network. Energy, 50:177-186.

Chavarria-Hernandez, J. C. and Pacheco-Catalán, D. E. (2014). Predicting the kinematic viscosity of \{FAMEs $\}$ and biodiesel: Empirical models. Fuel, 124(0):212 - 220.

Da Silva, I. N., Spatti, D. H., and Flauzino, R. A. (2010). Redes Neurais Artificiais para Engenharia e Ciências Aplicadas: curso prático. Artliber.

Dantas, M. B. (2006). Biodiesel blends: flow properties, thermal and oxidative stability during storage and monitoring. PhD thesis, Doctoral dissertation, $\mathrm{D}$. Sc. Thesis. Federal University of Paraíba. Brasil. Pag. 138.

Freitas, S. V., Segovia, J. J., Carmen Martín, M., Zambrano, J., Oliveira, M. B., Lima, A. S., and a.P. Coutinho, J. a. (2014). Measurement and prediction of high-pressure viscosities of biodiesel fuels. Fuel, 122:223-228.

García, M., Alba, J. J., Gonzalo, A., Sánchez, J. L., and Arauzo, J. (2013). Density of alkyl esters and its mixtures: A comparison and improvement of predictive models. Fuel, 103:232-238.

Hoekman, S. K., Broch, A., Robbins, C., Ceniceros, E., and Natarajan, M. (2012). Review of biodiesel composition, properties, and specifications. Renewable and Sustainable Energy Reviews, 16(1):143-169.

Jahirul, M. I., Senadeera, W., Brooks, P., Brown, R. J., Situ, R., Pham, P. X., and Masri, A. R. (2013). An Artificial Neutral Network (ANN) Model for Predicting Biodiesel Kinetic Viscosity as a Function of Temperature and Chemical Compositions. (December):1-6.

Kivevele, T. T., Mbarawa, M. M., Bereczky, a., Laza, T., and Madarasz, J. (2011). Impact of antioxidant additives on the oxidation stability of biodiesel produced from Croton Megalocarpus oil. Fuel Processing Technology, 92(6):1244-1248.

Knothe, G., Krahl, J., and Gerpen, J. V. (2010). Biodiesel Handbook. AOCS Press, 2nd edition.

Kohavi, R. et al. (1995). A study of cross-validation and bootstrap for accuracy estimation and model selection. In IJCAI, volume 14, pages 1137-1145.

Meng, X., Jia, M., and Wang, T. (2014). Neural network prediction of biodiesel kinematic viscosity at 313K. Fuel, 121:133-140.

Moser, B. R. (2009). Biodiesel production, properties, and feedstocks. In Vitro Cellular \& Developmental Biology - Plant, 45(3):229-266.

Nogueira, C. A. (2010). Densities and Viscosities of Binary Mixtures of Babassu Biodiesel Cotton Seed or Soybean Biodiesel at Different Temperatures. 55(11):5305-5310. 
Padhi, S. K. (2010). Preparation and Characterization of Biodiesel from Non-Edible Oils. PhD thesis, Doctoral dissertation, PhD thesis. National Institute of Technology, Rourkela.

Piloto-Rodríguez, R., Sánchez-Borroto, Y., Lapuerta, M., Goyos-Pérez, L., and Verhelst, S. (2013). Prediction of the cetane number of biodiesel using artificial neural networks and multiple linear regression. Energy Conversion and Management, 65:255-261.

Ramírez-Verduzco, L. F., Rodríguez-Rodríguez, J. E., and Jaramillo-Jacob, A. D. R. (2012). Predicting cetane number, kinematic viscosity, density and higher heating value of biodiesel from its fatty acid methyl ester composition. Fuel, 91(1):102-111.

Ramos, M. J., Fernández, C. M., Casas, A., Rodríguez, L., and Pérez, A. (2009). Influence of fatty acid composition of raw materials on biodiesel properties. Bioresource technology, 100(1):261-8.

Rezaee, M., Basri, M., Rahman, R. N. Z. R. A., Salleh, A. B., Chaibakhsh, N., and Masoumi, H. R. F. (2014). A multivariate modeling for analysis of factors controlling the particle size and viscosity in palm kernel oil esters-based nanoemulsions. Industrial Crops and Products, 52(0):506-511.

Saldana, D. A., Starck, L., Mougin, P., Rousseau, B., Ferrando, N., and Creton, B. (2012). Prediction of Density and Viscosity of Biofuel Compounds Using Machine Learning Methods. Energy \& Fuels, 26(4):2416-2426.

Sanford, S. D., White, J. M., Shah, P. S., Wee, C., Valverde, M. A., Meier, G. R., Group, R. E., and Report, C. (2009). Feedstock and Biodiesel Characteristics Report. pages $1-136$.

Santos, J. d. J. (2008). Biodiesel of Babassu: Thermal Verification, Oxidative and Binary Blends. PhD thesis, D. Sc. Thesis. Federal University of Paraí i ba, Brazil.

Sharon, H., Jayaprakash, R., Karthigai selvan, M., Soban kumar, D., Sundaresan, a., and Karuppasamy, K. (2012). Biodiesel production and prediction of engine performance using SIMULINK model of trained neural network. Fuel, 99:197-203.

Silva, L. N. d. C. (1998). Analise e sintese de estrategias de aprendizado para redes neurais artificiais. $\mathrm{PhD}$ thesis, Dissertação de mestrado. Universidade Estadual de Campinas. Brasil. Pag. 210. 